\title{
DUAL FEATURE EXTRACTION TECHNIQUES FOR IRIS RECOGNITION SYSTEM
}

\author{
Aro, T. O ${ }^{1}$, Jibrin, M. B ${ }^{3}$, Matiluko, O. E ${ }^{3}$, Abdulkadir, I. $\mathrm{S}^{4}$, Oluwaseyi, I. O ${ }^{5}$, \\ ${ }^{1}$ Department of Mathematical and Computing Sciences, KolaDiasi University, Ibadan, \\ Oyo State, Nigeria. \\ Email: taye.aro@koladiasiuniversity.edu.ng \\ ${ }^{2}$ Department of Computer Science, Federal University of Kashere, \\ Gombe State, Nigeria \\ Email:bashmjibrin@gmail.com \\ ${ }^{3}$ Landmark University Omu-Aran, Kwara State, Nigeria \\ Centre for System \& Information Services, Landmark University, Kwara State, Nigeria \\ Email:ssad@lmu.edu.ng \\ ${ }^{4}$ Department of Computer Science, Federal Polytechnic Offa, \\ Kwara State, Nigeria \\ Email:suhurat84@gmail.com \\ ${ }^{5}$ Department of Computer Science, Kogi State Polytechnic, Lokoja, \\ Kogi State, Nigeria \\ Email:oluwabukolabello@gmail.com \\ "Corresponding Author's Email:taiwo774@gmail.com
}

\begin{abstract}
The extraction of feature remains the significant phase in recognition system using iris. A successful recognition rate and reduction in classification time of two iris templates mostly depend on efficient feature extraction technique. This paper performs comparative analysis on two selected feature extraction techniques: Gabor Wavelet Transform (GWT) and Scale Invariant Feature Transform (SIFT). The developed system was evaluated with CASIA iris dataset. Performance evaluation of the system based on False Acceptance Rate (FAR), False Rejection Rate (FRR), Error Rate (ER) and accuracy produced different results of each technique. It was showed that the Gabor Wavelet Transform gave FAR of 0.9500 , FRR of $0.0750,92 \%$ of accuracy, and ER of $8 \%$ as compared with the SIFT technique which gave FAR of 0.900 , FRR of 0.0631 , ERR of $16.6 \%$ and $88.33 \%$ of accuracy. Finally, the results of comparative analysis showed that Gabor Wavelet Transform outperformed SIFT technique. From the results obtained, GWT is strongly recommended as a feature extraction method for the development of a robust iris authentication system.
\end{abstract}


Keywords: Feature Extraction, Gabor Wavelet Transform, Iris Recognition, Scale Invariant Feature Transform

\section{INTRODUCTION}

Iris recognition is a method that employs recognition using high resolution images of the humans' irises (Khotimah \& Juniati, 2018). Human's iris is a coloured and muscular portion within the pupil size, regulating amount of light that passes into eye (Shirke \& Gupta, 2013). This part of the eye is so much distinct, that is not possible to have two individual irises look the same, even for twins that are identical (Cappelli, Maio, Maltoni, Wayman, \& Jain, 2006). This technology is identified to be one of the most reliable biometrics due to its permanence, distinctiveness and non-invasive nature (Elgamal \& AlBiqami, 2013) The iris represents an annular point between white sclera and pupil, it has unusual structure which produces numerous interlacing of fine and unique features such as freckles, stripes, corona, furrows, crypts, rings, zigzag, collarets, radial, these are jointly referred to as iris texture (Pithadia \& Nimavat, 2015).

The Iris recognition is a dependable method that visually identify people when the imaging can be achieved at a close distances not exceeding 1 meter (Alaslani \& Elrefaei, 2018). This is quite suitable in areas where a huge database will require to be searched for recognition while ensuring there are minimal or no mis-matches (Daugman, 2009). Recently, the human iris has gained attention of biometric recognition system research and development (Pithadia \& Nimavat, 2015).

Features of an iris are distinguishable for different people, the unique features of the anatomical structure of iris makes it possible to differentiate individuals (Homayon, 2015). Iris pattern is unmatchable in which iris of each eye of the same person is entirely not the same. This makes the iris technology very important in biometric authentication system compared to other techniques like retina, finger, face, palm-print, odour and nose (Thyaneswaran \& Padma, 2014).

In iris recognition method, the extraction of features from region of iris is identified to be the most important phase, because the best features is required to be obtained with minimum classification and low execution time (Shah, Sharif, Raza, \& Azeem, 2013). Extraction of feature involves the transformation of original feature into a set of feature vector (Kumari \& Sharma, 2014). Unique features of the iris that encodes them into a code which is a set of mathematical parameters usually called a template, which is put in the database for matching an iris with those in the database. The most distinct features available in an image are extracted during feature extrtaction. It gives both local and global information of iris. Discriminated iris texture information must be extracted and encoded to have correct comparisons between iris templates. Complexity of feature extraction affects the complexity of program and processing speed of iris recognition system.

The extraction of features is considered to be the predominant aspect of iris recognition system due to the direct dependency of classification accuracy on the level of extracted features from iris region (Abhishree, Latha, Manikantan, \& Ramachandran, 2015). A number of extraction of feature techniques like Haar Wavelet Transform (HWT), Scale Invariant Feature Transform (SIFT), Local Binary Pattern, Gabor Wavelet Transform (GWT) have been used by researchers to extract iris texture. The extraction of features based on Gabor-filters have been widely employed in iris and fingerprint recognition (Khaladkar \& Ganorkar, 2012). The Gabor wavelet and SIFT have been proved to be the widely used techniques in iris recognition.

A Gabor wavelet is a filter bank with diverse orientations and scales, which is used to select textures of various densities and piece together for creation of iris image texture feature coding (Mude \& Patel, 2015). A SIFT is an effective technique for defining an image of local properties. In SIFT technique 
some important parts are not affected by lighting conditions, variation in scales and rotations (Gandhi \& Kulkarni, 2014). This approach is suitable in finding the salient, stable feature points in an image. In every image point, it gives features that describe a small region of image around the point. These features cannot be distorted by scale and rotation.

The performance of iris recognition system in terms of False Acceptance Rate (FAR) and False Recognition Rate (FRR) can also be affected by the feature extraction employed. For authentication system using iris scan, it necessary to actually ascertain how feature extraction will contribute to recognition rate. Nithya and Lakshmi (2016) stated clearly in the review study carried out on the different feature extraction techniques that of all of stages in iris recognition, the feature extraction algorithms plays a major role in improving the overall system performance in terms of accuracy and reliability.

The challenge of iris recognition system comes from the fact that it is not easy to pick the actual feature extraction technique that will give effective recognition system. Researchers have applied several techniques individually on iris datasets to develop recognition system, but much work has been not considered in comparing different extraction techniques. Looking at the unique characteristics of aforementioned feature extraction techniques. This paper performed comparative analysis on the two feature extraction techniques; GWT and SIFT.

\section{RELATED WORK}

Ahmed and Hamd (2018) developed an iris recognition system using two feature extraction techniques. The first approach applied the Fourier descriptors, which is based on transforming the uniqueness of iris texture to the frequency domain. The new frequency domain features was represented in iris-signature graph. The low spectrums defined the general description of iris pattern while the fine detail of iris is represented as high spectrum coefficients. The principle component analysis was employed as a second feature extraction. The system performance was evaluated by comparing the recognition results for fifty persons using the two methods. Three classifiers: Cosine, Euclidean, and Manhattan have been considered to evaluate the system performance for each approach separately. The classification results for Fourier descriptors on three classifiers gave $86 \%$ $94 \%$, and $96 \%$, versus $80 \%, 92 \%$, and $94 \%$ for principle component analysis. These results showed that Fourier descriptors method as feature extractor had better accuracy rate than principle component analysis.

Bansal, Agarwal and Sharma (2016) developed a correlation-based statistical technique for feature extraction technique between adjacent pixels. Hamming distance was applied for matching, to check the effectiveness of the system, false acceptance rate (FAR) and false rejection rate (FRR) with distance metric at different threshold were used. Experimental results obtained from using different set of statistical features of iris images showed better enhancement in equal error rate (ERR) when the statistical parameters for feature extraction were increased from three to six.

Patil \& Raghuveer (2015) proposed iris recognition system, the study applied several processes such as pre-processing, normalization, feature extraction and matching. A circular masking technique was introduced to normalize iris size. The two extraction of feature approaches; Bi-orthogonal Wavelet Transform (BWT) and Singular Value Decomposition (SVD) algorithms were employed for extraction of features, while a distance measure technique approach using euclidean was applied to 
find similarity between the iris images. Experimental findings showed major enhancements in accuracy of the developed system.

Ashwini, Mohammad and Fawaz (2015) came up with a multi-algorithmic technique in improving the iris recognition system accuracy. The study extracted features from the iris using different feature extraction algorithms, namely Local Pattern Quantization (LPQ), Local Binary Pattern (LBP), Gabor Filter, Haar, Daubechies8 and Daubechies16. The experimental results showed that Mutli-algorithms Iris Recognition System performed better than the unimodal system. The accuracy improvement offered by the new approach also reviewed that using more than two feature extraction algorithms in extracting the iris system might decrease the system performance. This is due to redundant features. The system presented a detailed description of the experiments.

Chirchi and Waghmare (2013) designed a system of feature extraction based method using a five level decomposition technique implemented with Haar, $\mathrm{db} 2$ and $\mathrm{db} 4$. The proposed system gave a comparative accuracy of $99.97 \%$, its speed was faster and equal error rate smaller than existing systems.

\section{METHODOLOGY}

The proposed iris recognition system involved so many different phases as shown in Figure 1. The first phase started with acquisition of iris images from Chinese Academy of Sciences' Institute of Automation (CASIA) database. The second phase is the localization process (find the outer radius of iris shapes), this process isolate iris region by locating pupil, two eyelids and eye lashes which cover some areas of the iris texture. After localization of the iris region in an eye image, normalization follows, due to the fact that irises of diverse people may be captured in different sizes the next step is to unwrap it into rectangular block of fixed dimensions. A Daugman Rubber Sheet method was used to fix dimensions. The next phase which is the real focus of this study, considered two extraction of feature methods; GWT and SIFT for extraction of feature texture from iris images. The different extracted features were passed to Hamming Distance for matching of iris image. 


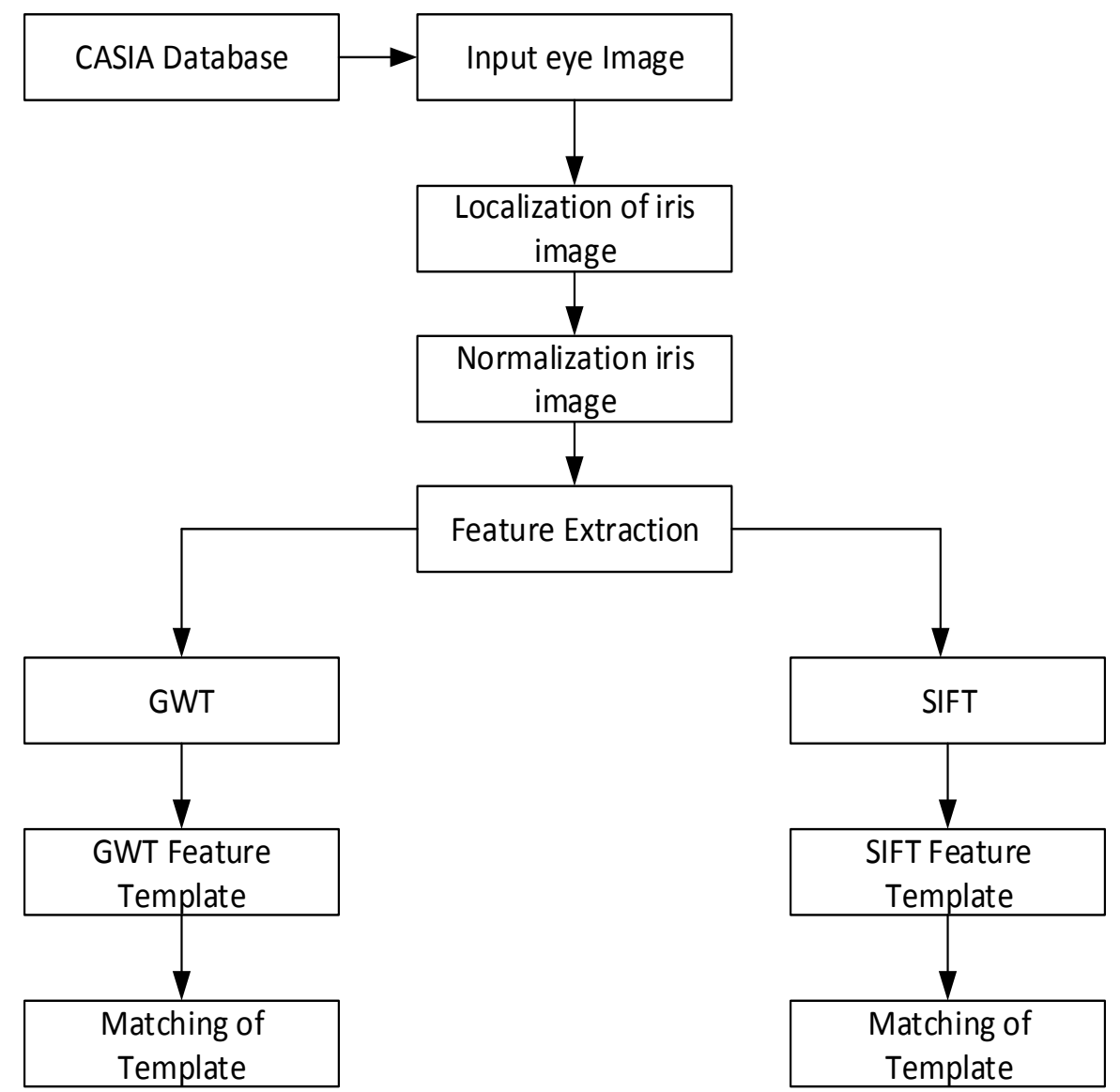

Figure 1: Framework of the Proposed System

\section{Iris Image Dataset}

Th iris image in CASIA database was used to evaluate the proposed system. Currently, the database belongs to one of the publicly available largest iris datasets. Figure 2 shows the sample of the database.
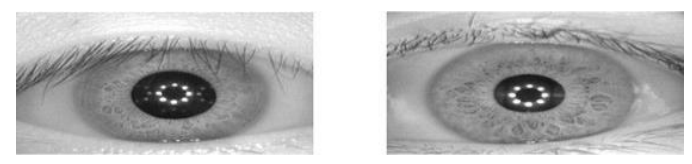

Figure 2: Sample of Iris image (CASIA Database)

\section{Setting of Parameters for Iris Database}

For each individual, the study used six data sets for training and for training, and two datasets which comprises of the left and right iris for testing. The training sets was sixty, testing sets was 24 and it consist of 10 class. Four out of the testing image were impostors the images were not found in the database. This phase allows the database parameters to be set before carrying out the experimental evaluation on proposed system as shown in Table 1. 
Table1: Parameters Setting of Iris Database

\begin{tabular}{cc}
\hline Parameters & Value \\
\hline Total number of training sets & 60 \\
Total number of Testing Sets & 24 \\
Total Iris Per subject & 6 \\
Total Number of Class (subjects) & 10 \\
Training Iris Per Class & 6 \\
Testing Iris Per Subject & 2 \\
Imposter & 4 \\
Total Iris Images & 88 \\
\hline
\end{tabular}

\section{Iris Image Processing}

The image processing techniques demarcates region of interest (ROI) from input image containing an eye. The image processing module contains two main tasks: Iris Segmentation and Iris Normalization. The Daughman's Integro-differential operator was employed to segment the iris portion accordingly. The normalization of the iris annular region to a rectangular region was achieved using rubber sheet of Daugman.

\section{Feature Extraction Algorithms}

The extraction of iris patterns was done using two iris feature extraction algorithms: Gabor Wavelet Transform and SIFT.

\section{(i) SIFT Algorithm}

The four major steps for computation SIFT method are Scale Space Extrema Detection, Keypoint Localization, orientation Assignment and Keypoint Descriptor, which are detailed as follows:

\section{Step 1: Detection of Scale Space Extrema}

The entire scale and image location are identified. Gaussian function difference is applied to find the keypoints from annular iris image. An input iris image $\mathrm{I}(\mathrm{x}, \mathrm{y})$ of scale space is a function of $\mathrm{L}(\mathrm{x}, \mathrm{y}, \sigma)$ which is convolved with Gaussian kernel $\mathrm{G}(\mathrm{x}, \mathrm{y}, \sigma)$ and given as:

$$
\begin{aligned}
& \mathrm{G}(\mathrm{x}, \mathrm{y}, \sigma)=\frac{1}{2 \pi \sigma^{2}} e^{\frac{\left(-x^{2}-y^{2}\right)}{2 \sigma^{2}}} \\
& \mathrm{~L}(\mathrm{x}, \mathrm{y}, \sigma)=\mathrm{G}(\mathrm{x}, \mathrm{y}, \sigma) * \mathrm{I}(\mathrm{x}, \mathrm{y})
\end{aligned}
$$

Where $*$ is the convolution operation in $\mathrm{x}$ and $\mathrm{y} . \sigma$ defines the width of the Gaussian filter. The Gaussian images differences are calculated from the difference of two nearby scales separated by a constant multiplicative factor $\mathrm{k}$. 


$$
\mathrm{D}(\mathrm{x}, \mathrm{y}, \sigma)=\mathrm{L}(\mathrm{x}, \mathrm{y}, \mathrm{k} \sigma)-\mathrm{L}(\mathrm{x}, \mathrm{y}, \sigma)
$$

\section{Step 2: Keypoint Localization}

The comparison of two nearby pixels gives candidate keypoint. The next step performs the detailing to the candidate keypoint to remove the regions which are badly localized. The Gaussian difference finds regions of interest using local maxima and minima.

\section{Step 3: Orientation Assignment}

This phase gives the individual selected keypoints orientation which leads to invariance of rotation. The gradient direction and the individual keypoints magnitude are collected and the most prominent orientation is considered as the keypoint in that region. The keypoint orientation is obtained by computing the gradient orientation histogram.

\section{Step 4: Keypoint Descriptor Computation}

The computation of feature descriptor is set as an orientation histogram on $4 \times 4$ pixel neighbourhoods. For $16 \times 16$ neighbourhood around the keypoint was taken, shared into 16 sub-blocks of $4 \times 4$ size. For each sub-block, 8-bin orientation histogram was produced. So a total of $4 \times 4 \times 8=128$ bins values. These 128 numbers give the feature vector and the keypoint was uniquely identified by this feature vector.

\section{(ii) Gabor Wavelet Transform Algorithm}

The Gabor-filters for extraction of features are described with following steps:

\section{Step 1: Transformation of Image}

The inferior and superior cones of iris are removed. The size differences of the iris are recompensed using an image polar sampling, obtaining $\mathrm{J}$ as a result. Transformation equation is as follows:

$$
\mathrm{J}(\rho, \phi)=I_{E}\left(x_{0}+r \cos \theta, y_{0}+r \sin \theta\right)
$$

Where

$$
\begin{aligned}
& \mathrm{r}=r_{i}+(\rho-1) \Delta_{r}, \forall \rho \in N: \rho \leq \frac{r_{e}-r_{i}}{\Delta_{r}} \\
& \mathrm{v}= \begin{cases}\frac{-\pi}{4}+(\Phi-1) \times \Delta_{\theta} & \text { if } \phi \leq \frac{\pi}{2 \Delta_{0}} \\
\frac{3 \pi}{4}+(\phi-1) \times \Delta_{\theta} & \text { if } \phi>\frac{\pi}{2 \Delta_{0}}\end{cases}
\end{aligned}
$$

where

$I_{E}$ denotes the iris image gray level with the sclera and pupil extracted $r_{i}$ and $r_{e}$ are the inner and outer radius. $\left(x_{0} y_{0}\right)$ is the pupil centre, and $\Delta_{r}$ and $\Delta_{0}$, are the magnitude and angle sample intervals.

Step 2: Convolute Gabor filter with iris image

Imaginary part of Gabor filter $\mathrm{J}$ is weighted with orientations $\left(0, \frac{\pi}{4}, \frac{\pi}{2}\right.$ and $\left.\frac{3 \pi}{4}\right)$. This is computed by the division of image in squared section with following equation used:

$\mathrm{C}(\mathrm{i} \mathrm{j})=\sum_{x=1}^{N} \sum_{y=1}^{M} j\left(i+x-\frac{N}{2}, j+y-\frac{M}{2}\right) \times g\left(x, y, \varphi_{k}, \lambda\right)$

Where 


$$
g\left(x, y, \varphi_{k}, \lambda\right)=\exp \left\{\exp -\left(\frac{x \cos \psi_{k}+y \sin \psi_{k}}{\sigma_{x}^{2}}\right)^{2}+\left(-\left(\frac{-x \sin x_{\varphi}}{\sigma_{y^{2}}{ }^{2}}\right)\right\} \mathrm{X} \sin \left\{\frac{2 \pi\left(x \cos \varphi_{k}+y \sin \varphi_{k}\right)}{\lambda}\right\}\right.
$$

The filter dimension is $\mathrm{N} \times \mathrm{M},(\mathrm{i}, \mathrm{j})$ is the centre of each section and $x, \lambda, \sigma_{x}, \sigma_{y}$ are filter parameters.

\section{Classification of Iris Images using Hamming Distance}

The extracted portion from feature extraction techniques was used as iris template by observing the feature vector of each iris image as the portion to formulate the system database. For classification, Hamming distance (HD) was used to classify iris images into matched or mismatched. This measure shows the number of bits that are the same in two bit patterns. With the application of HD of two bit patterns, a conclusion is drawn as which of the two patterns produce from different irises or from the same one. In comparing the bit patterns $\mathrm{X}$ and $\mathrm{Y}$, the HD is given in Equation (9)

$$
\mathrm{HD}=\frac{1}{N} \sum_{J-1}^{N} X_{j}(X O R) Y_{j}
$$

Where $X O R$ is sum of the exclusive OR between $\mathrm{X}$ and $\mathrm{Y}$ ) over $\mathrm{N}$ and $\mathrm{N}$ is the amount of bits in the bit pattern.

\section{Performance Evaluation Metrics of the System}

The system was evaluated with the following evaluation parameters; True Positive (TP), True Negative (TN), False Negative (FN), False Positive (FP). From this, the False Acceptance Rate, False Rejection Rate, Error Rate and Accuracy then computed by applying the following equations.

$$
\begin{aligned}
\text { False Acceptance Rate } & =\frac{\text { No of Accepted Imposters }}{\text { Total No of Imposters Assessed }} \\
= & \frac{F P}{F P+T N} \\
\text { False Rejection Rate } & =\frac{\text { No of Reje ted Genuine }}{\text { Total } \text { No of Geniune Assessed }} \\
& =\frac{F N}{T P+F N} \\
\text { Error Rate } & =\frac{F P+F N}{T P+T N+F P+F N} \times 100 \\
\text { Accuracy } & =\frac{T P+T N}{T P+T N+F P+F N} \times 100
\end{aligned}
$$




\section{RESULTS AND DISCUSSION}

The experimental results of the developed system are described in this section. After the iris image processing, the processed iris images were passed separately to two feature extraction techniques; GWT and SIFT in order to obtain iris feature templates. The outputs of feature extraction methods is given in the following subsections.

\section{Results of GWT Feature Extraction}

The sample for template and mask of extracted feature of GWT is shown in Figure 2 (a) and 2 (b). The classification (matching) of GWT feature templates were achieved using hamming distance measure with threshold value set to $\leq 0.3732$ as shown in Table 2 and Table 3 for the iris images in the database and impostors.

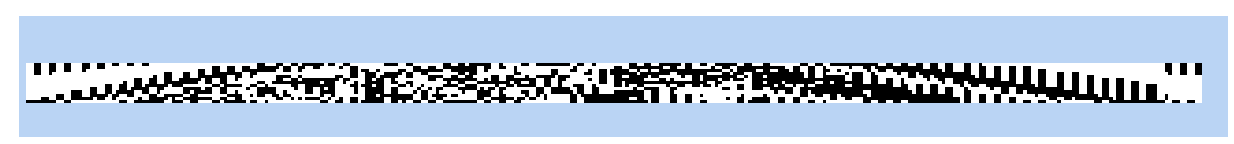

Figure 2(a): Template

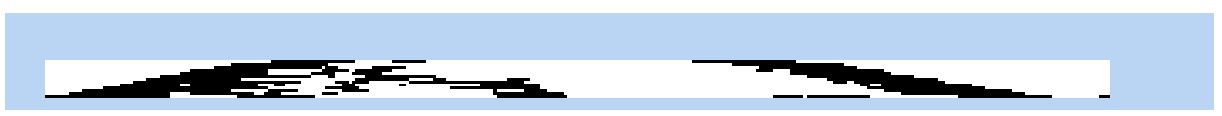

Figure 2(b): Mask 
Table 2: Result of Classification of Extracted Iris Features Using Gabor Wavelet Transform

\begin{tabular}{|c|c|c|c|c|c|}
\hline $\begin{array}{c}\text { Serial } \\
\text { number }\end{array}$ & Class & $\begin{array}{c}\text { Index of image } \\
\text { recognized }\end{array}$ & $\begin{array}{c}\text { Hamming distance } \\
(\mathbf{m})\end{array}$ & $\begin{array}{l}\text { Testing Time } \\
\text { (secs) }\end{array}$ & $\begin{array}{l}\text { Matching } \\
\text { Status }\end{array}$ \\
\hline 1 & 1 & 1 & 0.2541 & 5.5567 & Matched \\
\hline 2 & 1 & 3 & 0.2279 & 5.4991 & Matched \\
\hline 3 & 2 & 2 & 0.1347 & 5.5254 & Matched \\
\hline 4 & 2 & 2 & 0.1620 & 5.2367 & Matched \\
\hline 5 & 3 & 1 & 0.2541 & 5.3485 & Matched \\
\hline 6 & 3 & 3 & 0.2279 & 5.4863 & Matched \\
\hline 7 & 4 & 2 & 0.1347 & 6.4831 & Matched \\
\hline 8 & 4 & 2 & 0.1620 & 6.3880 & Matched \\
\hline 9 & 5 & 2 & 0.1620 & 6.3616 & Matched \\
\hline 10 & 5 & 1 & 0.2541 & 4.9278 & Matched \\
\hline 11 & 6 & 1 & 0.2541 & 4.5416 & Matched \\
\hline 12 & 6 & 1 & 0.3541 & 5.4471 & Matched \\
\hline 13 & 7 & 3 & 0.2279 & 4.6760 & Matched \\
\hline 14 & 7 & 2 & 0.3347 & 5.2055 & Matched \\
\hline 15 & 8 & 2 & 0.3620 & 5.4618 & Matched \\
\hline 16 & 8 & 1 & 0.3541 & 5.4533 & Matched \\
\hline 17 & 10 & 3 & 0.3779 & 5.4833 & Mismatched \\
\hline 18 & 9 & 2 & 0.1347 & 5.4022 & Matched \\
\hline 19 & 10 & 2 & 0.1620 & 5.0313 & Matched \\
\hline 20 & 1 & 2 & 0.1620 & 5.1632 & Matched \\
\hline
\end{tabular}


From Table 2, the serial number, class, index of image classified, hamming distance and testing-time and matching status are shown. The classification is positive for any image with threshold of $\leq$ 0.3732. Out of 20 iris images employed from CASIA dataset, iris image in class 10, index image 3, hamming distance value of 0.3779 was mismatched during classification phase.

Table 3: Result of Classification For Extracted Features Using Gabor Wavelet Transform (Impostor)

\begin{tabular}{ccccc}
\hline Serial number & class & $\begin{array}{c}\text { Index of image } \\
\text { recognized }\end{array}$ & $\begin{array}{c}\text { Hamming } \\
\text { distance }\end{array}$ & $\begin{array}{c}\text { Testing Time } \\
\text { (secs) }\end{array}$ \\
\hline 21 & No Class & No Image & No Value & 5.1822 \\
22 & No Class & No Image & No Value & 5.8061 \\
23 & 3 & 1 & 0.1347 & 5.0359 \\
24 & No Class & No Image & No Value & 6.2378 \\
\hline
\end{tabular}

Table 3 shows the classification of iris images for impostors (iris images not contained in the database), only iris image in class 3, index of image 1, hamming distance value of 0.1347 was wrongly classified as authentic iris image. Where there is no any displace for iris of no hamming distance value.

\section{Results of SIFT Feature Extraction}

The sample for template and mask of extracted feature of SIFT is shown in Figure 3(a) and 3(b). The SIFT feature templates were matched using Hamming Distance measure with threshold value set to $\leq 0.3732$ as shown in Table 4 and Table 5 .

\section{W}

Figure 3(a): Template

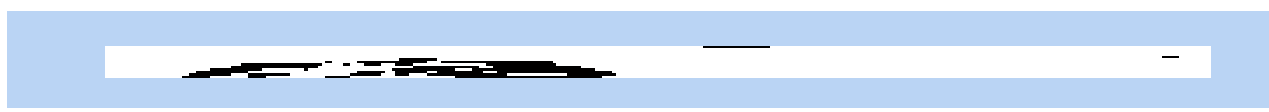

Figure 3(b): Mask 
Table 4: Result of Extracted Features Using SIFT

\begin{tabular}{|c|c|c|c|c|c|}
\hline $\begin{array}{l}\text { Serial } \\
\text { number }\end{array}$ & Class & $\begin{array}{l}\text { Index of } \\
\text { image } \\
\text { recognized }\end{array}$ & $\begin{array}{l}\text { Hamming } \\
\text { distance }\end{array}$ & $\begin{array}{l}\text { Testing time } \\
\text { (secs) }\end{array}$ & $\begin{array}{c}\text { Matching } \\
\text { Status }\end{array}$ \\
\hline 1 & 1 & 1 & 0.3421 & 6.7894 & Matched \\
\hline 2 & 1 & 3 & 0.3579 & 5.7869 & Matched \\
\hline 3 & 2 & 2 & 0.2277 & 6.3241 & Matched \\
\hline 4 & 2 & 2 & 0.2150 & 5.6765 & Matched \\
\hline 5 & 3 & 1 & 0.3641 & 6.0897 & Matched \\
\hline 6 & 3 & 3 & 0.3467 & 6.9845 & Matched \\
\hline 7 & 4 & 2 & 0.2456 & 5.7685 & Matched \\
\hline 8 & 4 & 2 & 0.2520 & 6.7876 & Matched \\
\hline 9 & 5 & 2 & 0.2345 & 7.6765 & Matched \\
\hline 10 & 5 & 1 & 0.3478 & 5.6876 & Matched \\
\hline 11 & 6 & 1 & 0.3675 & 6.7576 & Matched \\
\hline 12 & 6 & 1 & 0.3867 & 6.7809 & Mismatched \\
\hline 13 & 7 & 3 & 0.3324 & 4.9084 & Matched \\
\hline 14 & 7 & 2 & 0.2543 & 6.1111 & Matched \\
\hline 15 & 8 & 2 & 0.2765 & 6.7856 & Matched \\
\hline 16 & 8 & 1 & 0.3946 & 5.1123 & Mismatched \\
\hline 17 & 10 & 3 & 0.3452 & 4.3453 & Matched \\
\hline 18 & 9 & 2 & 0.2435 & 6.7675 & Matched \\
\hline 19 & 10 & 2 & 0.2345 & 4.7688 & Matched \\
\hline 20 & 1 & 2 & 0.2790 & 3.6756 & Matched \\
\hline
\end{tabular}


From Table 4, the serial number of 12 and 16 (in class 6 and 8) with hamming distance values of 0.3867 and 0.3946 were mismatched during classification. The level of misclassification of SIFT feature-based iris recognition is higher than the Gabor Wavelet Transform.

Table 5: Result of Classification of Extracted Features Using SIFT (Impostor)

\begin{tabular}{ccccc}
\hline Serial number & Class & $\begin{array}{c}\text { Index of image } \\
\text { recognized }\end{array}$ & Hamming distance & $\begin{array}{c}\text { Testing time } \\
\text { (secs) }\end{array}$ \\
\hline $\mathbf{2 1}$ & No Class & No Image & No Value & 7.6556 \\
$\mathbf{2 4}$ & No Class & No Image & No Value & 6.5654 \\
$\mathbf{2 2}$ & 3 & 0.1347 & 0.1347 & 6.4532 \\
$\mathbf{2 3}$ & 2 & 0.298 & 0.298 & 7.6763 \\
\hline
\end{tabular}

Table 5, the serial number of 22 and 23 (in class 3 and 2) with hamming distance values of 0.1347 and 0.298 were mismatched during classification. The level of impostor for SIFT feature-based iris recognition is higher than the Gabor Wavelet Transform.

\section{Result of Comparative Analysis of the Dual Feature Extraction for Iris Recognition}

The comparative analysis of feature extraction methods was analyzed using the experimental results of evaluation metrics as shown in Table 6.

Table 6: Comparative Analysis of the Dual Feature Extraction Technique for Iris Recognition

\begin{tabular}{ccc}
\hline Evaluation Metrics & GWT & SIFT \\
\hline False Acceptance Rate & 0.9500 & 0.9000 \\
False Rejection Rate & 0.0750 & 0.0631 \\
Error Rate & $8 . \%$ & $16.67 \%$ \\
Accuracy & $92 \%$ & $83.33 \%$ \\
\hline
\end{tabular}

\section{CONCLUSION}

The iris feature extraction is a significant step of iris recognition system. Several feature extraction techniques have been proposed by researchers for the extraction of iris features template from iris images. This paper applied two iris feature extraction methods: Gabor Wavelet Transform (GWT) and Scale Invariant Feature Transform (SIFT) in which a comparative analysis was done on these 
extraction methods. The evaluation metrics based on False Acceptance Rate (FAR), False Rejection Rate (FRR), Error Rate and accuracy gave distinct results of each method. It was revealed that the Gabor Wavelet Transform gave FAR of 0.9500 , FRR of $0.0750,92 \%$ of accuracy, and ERR of $8 \%$ as compared with SIFT which gave FAR of 0.900 , FRR of 0.0631 , ER of $16.6 \%$ and $88.33 \%$ of accuracy. The overall experimental results showed that Gabor Wavelet Transform performed better than SIFT technique. Also, fusion of two or more feature extraction techniques should be considered for future work.

\section{REFERENCES}

Abhishree, T. M., Latha, K., Manikantan, \& Ramachandran, S. (2015). Face Recognition Using Gabor Filter Based Feature Extraction with Anisotropic Diffusion as a Pre-processing Technique 2 . International Conference on Advanced Computing Technologies and Applications, 45, 312-321.

Ahmed, S. K., \& Hamd, M. (2018). Biometric System Design for Iris Recognition Using Intelligent Algorithms. International Journal of Modern Education and Computer Science, 3, 9-16. http://doi.org/10.5815/ijmecs.2018.03.02

Alaslani, M. G \& Elrefaei, L. A. (2018). Convolutional Neural Network Based Feature Extraction For Iris Recognition. International Journal of Computer \& Information Technology, 10(2), 65-78. http://doi.org/10.5121/ijcsit.2018.10206

Ashwini, M. B., Mohammad, I. \&, \& Fawaz, A. (2015). Evaluation of Iris Recognition System on Multiple Feature Extraction Algorithms and its Combinations. International Journal of Computer Applications Technology and Research, 4(8), 592-598. http://doi.org/10.7753/IJCATR0408.1002

Bansal, A., Agarwal, R., \& Sharma, R. K. (2016). Statistical feature extraction based iris recognition system. Sadhana, 41(5), 507-518. http://doi.org/10.1007/s12046-016-0492-9

Cappelli, R., Maio, D., Maltoni, D., Wayman, J. L., \& Jain, A. K. (2006). Performance evaluation of fingerprint verification systems. IEEE Transactions on Pattern Analysis and Machine Intelligence, 28(1), 3-17. http://doi.org/10.1109/TPAMI.2006.20

Chirchi, V. R. E., \& Waghmare, L. M. (2013). Feature Extraction and Pupil Detection Algorithm Used for Iris Biometric Authentication System, 6(6), 141-160. http://doi.org/10.14257/ijsip.2013.6.6.14

Daugman, J. (2009). How Iris Recognition Works. The Essential Guide to Image Processing, 14(1), 715-739. http://doi.org/10.1016/B978-0-12-374457-9.00025-1

Elgamal, M. \& Al-Biqami, N. (2013). An Efficient Feature Extraction Method for Iris Recognition Based on Wavelet Transformation. International Journal of Computer and Information Technology, 02(03), 521-527.

Gandhi, K. M., \& Kulkarni, P. R. H. (2014). Sift Algorithm for Iris Feature Extraction. Global Journal of Computer Science and Technology Graphics \& Vision, 14(3), 0-6.

Homayon, S. (2015). Iris Recognition for Personal Identification Using LAMSTAR Neural Network. 
International Journal of Computer Science and Information Technology, 7(1), 1-8. http://doi.org/10.5121/ijcsit.2015.7101

Khaladkar, M., \& Ganorkar, S. R. (2012). Comparative Analysis for Iris Recognition. International Journal of Engineering Rsearch \& Technology, 1(4), 1-6.

Khotimah, C. \& Juniati, D. (2018). Iris Recognition Using Feature Extraction of Box Counting Fractal Dimension Iris Recognition Using Feature Extraction of Box Counting Fractal Dimension. In IOP Conference Series (pp. 1-6).

Kumari, Y., \& Sharma, R. (2014). Iris Recognition System using Gabor Filter \& Edge Detection. International Journal on Recent and Innovation Trends in Computing and Communication, 2(8), 2265-2269.

Mude, R \& Patel, M. R. (2015). Gabor Filter for Accurate IRIS Segmentation Analysis. International Journal of Innovations in Engineering and Technology, 6(1), 148-153.

Nithya, A \& Lakshmi, C. (2016). Feature Extraction Techniques For Recognition of Iris Images : A Review. iIJCTA, 9(28), 88-92.

Patil, M., \& Raghuveer, K. (2015). RESEARCH ARTICLE SVD and DWT Based Iris Recognition Using Beagleboard-xM. International Journal of Computer Science and Mobile Computing, 4(4), $532-540$.

Pithadia, N. J., \& Nimavat, V. D. (2015). A Review on Feature Extraction Techniques. International Journal of Engineering Research \& Technmology, 1(3), 1263-1268.

Shah, J. H., Sharif, M., Raza, M., \& Azeem, A. (2013). A Survey : Linear and Nonlinear PCA Based Face Recognition Techniques. International Arab Journal of Information Technology, 10(6), $536-545$.

Shirke, S. D., \& Gupta, D. (2013). IRIS RECOGNITION USING GABOR. International Journal of Computer Technology \& Application, 4(1), 1-7.

Thyaneswaran, B. \& Padma, S. (2014). Analysis of Gabor Filter Parameter for Iris Feature Extraction. Internationa Journal of Advanced Computer Technology, 3(5), 45-48. 